\title{
Influência do aleitamento materno na hospitalização de menores de dois anos no estado de Pernambuco, Brasil, em 1997 e 2006
}

\author{
The influence of breastfeeding in the hospitalization of children \\ under two years of age in the State of Pernambuco, Brazil, \\ in 1997 and 2006
}

Thaysa Thatyana Aragão Guerra Mota ${ }^{1}$

Maria de Fátima Costa Caminha ${ }^{1}$

José Natal Figueiroa ${ }^{1}$

Pedro Israel Cabral de Lira ${ }^{2}$

Malaquias Batista Filho ${ }^{1}$

${ }^{1}$ Diretoria de Pesquisa, Instituto de Medicina Integral Prof. Fernando Figueira, Fiocruz. R. Coelhos 300, Coelhos. 50070-550 Recife PE Brasil.

thaysamota@yahoo.com.br

${ }^{2}$ Departamento de

Nutrição, Centro de

Ciências da Saúde,

Universidade Federal de

Pernambuco.

\begin{abstract}
The scope of this paper was to evaluate the influence of breastfeeding and other factors on the incidence of hospitalization and the frequency and main causes of hospitalization in children under two years of age in the State of Pernambuco. It is a cross-sectional, descriptive-analytical study using secondary data obtained from the II and III State Health and Nutrition Surveys (PESN) carried out in 1997 and 2006, respectively. A sample of 1503 infants under two years of age was studied. Pearson's Chi-square test was used to analyze bivariate associations and Fisher's exact test for comparison of risk of hospitalization in the two years of observation and Poisson regression for the adjustment of the multivariate model of hospitalizations in the last survey. In the two years analyzed, there was no statistically significant difference in the percentage of hospitalizations of children. The lower risk of hospitalization remained for those who were breastfed exclusively. Regarding to environmental, socioeconomic, demographic characteristics and health assistance, several factors have evolved favorably in the period assessed. In the final model, only the weight/ length ratio (low weight category) remained associated with the risk of hospitalization in children under two years of age factors.
\end{abstract}

Key words Breastfeeding, Hospitalization, Child health, Epidemiology
Resumo Objetivou-se avaliar a influência do aleitamento materno e de outros fatores na ocorrência de hospitalizações, bem como a frequência e principais causas de internações em menores de dois anos, no estado de Pernambuco, Brasil. Estudo transversal, descritivo-analítico, utilizando dados secundários obtidos dos bancos das II e III Pesquisas Estaduais de Saúde e Nutrição (PESN), realizadas nos anos de 1997 e 2006, respectivamente, totalizando uma amostra de 1503 menores de dois anos. Utilizou-se o teste chi-quadrado de Pearson, para analisar associações bivariadas, o Exato de Fisher, para comparação de risco de hospitalização nos dois anos observados, e a regressão de Poisson, para o ajustamento de modelo multivariado dos fatores condicionantes da internação no último inquérito. Nos dois anos analisados, não houve diferença estatisticamente significante no percentual de hospitalizações das crianças. $O$ menor risco de internação permaneceu para os que mamaram exclusivamente. No que se refere às características ambientais, socioeconômicas, demográficas e de assistência à saúde, vários fatores evoluíram favoravelmente no período avaliado. No modelo final, apenas o indice peso/ comprimento (categoria baixo peso) permaneceu associado aos fatores de risco de hospitalização de menores de dois anos.

Palavras-chave Aleitamento materno, Hospitalização, Saúde da criança, Epidemiologia 


\section{Introdução}

Provavelmente as práticas e os juízos de valores relacionados com a questão do aleitamento materno representam um dos capítulos mais conflitivos da história do comportamento alimentar do homem ${ }^{1}$. Sendo um evento essencialmente biológico, a ponto de caracterizar um segmento variado de seres hierarquicamente representados na escala biológica (os mamíferos), as práticas de amamentação passaram a sofrer influências marcantes no homem e animais domésticos submetidos a sua ação. Este processo de intervenção sobre a conduta natural da amamentação resultou em comportamentos variados e em manifestações atípicas, entre as quais figuram situações gravemente adversas em relação à saúde ${ }^{2}$.

$\mathrm{Na}$ realidade, interesses econômicos (notadamente mercadológicos), princípios religiosos, tradições ou modismos culturais, processos políticos e fatores psicossociais, além do acervo considerável de conhecimentos científicos e tecnológicos, têm exercido marcantes repercussões sobre as práticas de amamentação, notadamente a partir da Revolução Industrial, no advento do século 19, acentuando-se após a Segunda Guerra Mundial, em meados do Século XX1. Desde então, o acúmulo de evidências, experimentais clínicas e epidemiológicas sob variadas circunstâncias, têm consolidado a compreensão de que, nos primeiros meses de vida, o aleitamento materno constitui, sob o ponto de vista de saúde, a melhor alternativa para a alimentação das crianças, tendo em vista seu papel sobre o estado de nutrição, proteção imunológica e desenvolvimento neuropsíquico das crianças ${ }^{3}$. Essas vantagens são cruciais na prevenção da morbimortalidade nos primeiros meses e anos de vida, estendendo-se à adolescência, vida adulta e até à senescência, como fator de redução de riscos de doenças crônicas não transmissíveis (DCNT), como o diabetes tipo 2, a obesidade, as doenças cardiovasculares, dislipidemias, muitos processos neoplásicos e afecções osteoarticulares ${ }^{4-7}$.

Mas, indiscutivelmente, os efeitos protetores mais notáveis e mais imediatos se aplicam às crianças nos primeiros seis meses, e, secundariamente, nos primeiros dois anos de vida, quando $o$ aleitamento materno representa um diferencial de primeira ordem em relação à ocorrência de doenças, duração e gravidade de sua evolução e complicações, principalmente nos países em desenvolvimento e nas camadas socioeconômicas de baixas condições de vida ${ }^{8}$. Neste sentido, o registro de morbidades, de demandas nos ser- viços de saúde, incluindo hospitalizações, pode figurar como um campo de interesse prioritário, seja como abordagem de pesquisas, seja como objeto de preocupações de políticas e programas de saúde. Esta relevância pode ser destacada no reconhecimento de que o aleitamento materno é considerado uma das três estratégias mais importantes, por seu baixo custo e elevada eficácia, para a promoção da saúde das crianças nos primeiros 24 meses de vida'. Embora universal, este reconhecimento e sua validação são particulares para cada contexto histórico, socioeconômico, ambiental e condições de assistência à saúde, justificando, assim, a pertinência, oportunidade e relevância de avaliações periódicas, relativizando seus resultados e sua abordagem analítica na realidade contextual de cada país, região ou comunidade $^{10}$.

É, portanto, nesta perspectiva que se propõe o presente estudo, referenciado no possível papel do aleitamento materno como fator de diferenciação do risco de hospitalização de crianças nos primeiros dois anos de vida, no Estado de Pernambuco, numa abordagem de tendência temporal e, num segundo aspecto, de compreensão analítica de outros fatores condicionantes de internação hospitalar.

\section{Contexto e método de estudo}

Trata-se de um estudo de abordagem quantitativa, observacional, transversal, descritivo com componentes analíticos. Baseia-se nos dados dos bancos da II e da III Pesquisa Estadual de Saúde e Nutrição (PESN), realizadas no Estado de Pernambuco, respectivamente nos anos de 1997 e 2006.

Dando sequência a um inquérito inicial realizado em $1991^{11}$, estes dois últimos estudos objetivaram atualizar e ampliar o diagnóstico da situação de saúde, nutrição, alimentação, condições socioeconômicas e serviços de assistência da população do estado, com ênfase no grupo materno-infantil, distribuindo-se em três estratos geoeconômicos: Região Metropolitana do Recife (RMR), Interior Urbano (IU) e Interior Rural (IR). Considerou-se como unidade de estudo a família com crianças menores de cinco anos.

Na II PESN-PE/ 1997, a amostragem do tipo probabilística repetiu a sequência de estágios dos sorteios nos municípios da PESN de 1997 em três estágios, através de sorteio sequencial de município, setor censitário e domicílio. No total, 18 municípios fizeram parte da amostra, seleciona- 
dos por sorteio aleatório, considerando as mesmas cidades participantes do primeiro Inquérito, tendo em vista a conveniência de comparação dos resultados nos diferentes anos. A amostra foi composta por 2.078 crianças menores de cinco anos, sendo 737 da Região Metropolitana do Recife, 687 do interior urbano e 654 no interior rural $^{12}$

$\mathrm{Na}$ Pesquisa Estadual do ano 2006, para a composição da amostra, repetiu-se a sequência de estágios de sorteios dos municípios da PESN de 1997. Participaram do estudo 852 crianças do setor urbano (RMR: 431 e interior urbano: 421 ) e 798 do setor rural, as quais foram unificadas num valor total de 1650 menores de cinco anos ${ }^{13}$.

Para o artigo aqui relatado, foi construído um arquivo ad hoc com as variáveis necessárias para responder aos objetivos específicos do estudo, demandando a recriação e recodificação de algumas variáveis a partir dos bancos originais. Foram excluídas as crianças que não dispunham de informações completas e consistentes sobre hospitalizações e práticas de aleitamento materno. Utilizou-se a classificação da OMS ${ }^{14}$ para os tipos de aleitamento (misto, predominante e exclusivo), considerando como segunda alternativa de referência, o aleitamento exclusivo por quatro meses ou mais.

A amostra e os procedimentos descritivos e analíticos utilizados foram enfocados em três eixos metodológicos: a) caracterização biológica das crianças (menores de dois anos) e das variáveis socioeconômicas, demográficas e obstétricas de suas mães; b) identificação e comparação da frequência e causas de hospitalizações na amostra estudada, analisando a influência da duração e tipologias do aleitamento materno no risco destas internações; e c) avaliação da contribuição comparativa de outras co-variáveis na ocorrência de hospitalização de crianças no ano de 2006, por meio de análises estatísticas uni e multivariadas.

O tamanho amostral foi calculado por meio de recurso específico do Software Epi-Info versão 7.1. Aceitando-se uma ocorrência de $10 \%$ de expectativa de hospitalizações ${ }^{15}$, com um erro de $2,5 \%$, obteve-se o valor de 552 crianças para cada ano da pesquisa, adotando um nível de confiança de $95 \%$ e poder estatístico de $80 \%$. No final, a amostra total foi composta por 1.503 crianças menores de dois anos, sendo 870 do segundo inquérito e 633 crianças do terceiro.

Para a análise dos dados, utilizou-se o programa Stata versão 12.0. Os resultados apresentados em tabelas foram resumidos através de frequência absoluta e percentual. Para a comparação estatística das características amostrais referentes às variáveis descritivas de 1997 e 2006 foi utilizado o teste chi-quadrado de Pearson. O teste exato de Fisher foi aplicado no sentido de avaliar o risco de hospitalizações segundo o tipo e a duração do aleitamento nos dois diferentes anos. A análise dos fatores associados à hospitalização das crianças, realizada através da regressão de Poisson, estimando-se as razões de prevalência (RP) brutas e ajustadas. Seguiram para a análise ajustada as variáveis que apresentaram um valor $\mathrm{p}<0,20 \mathrm{e}$ permaneceriam no modelo final se caso o valor $\mathrm{p}<0,05$.

O presente estudo foi submetido aos padrões requeridos pela Resolução 466/12 $2^{16}$, sendo aprovado pelo Comitê de Ética em Pesquisa do Instituto de Medicina Integral Prof. Fernando Figueira (IMIP).

\section{Resultados}

A Tabela 1 mostra as características biológicas das crianças, variáveis socioambientais e demográficas maternas, antecedentes obstétricos e dados referentes ao acesso aos serviços e ações de saúde, realizando-se análise de significância estatística destas variáveis após comparação nos dois anos pesquisados. A maioria das variáveis (16 das 23 avaliadas) apresentou diferenças estatisticamente significativas, considerando o valor $\mathrm{p}<0,05 \mathrm{e}$ IC $95 \%$, revelando diversos aspectos nas práticas de aleitamento materno, nas condições de vida e saúde da população pernambucana.

No grupo das "características relativas às crianças", o percentual de menores que nunca mamaram diminuiu de 1997 para 2006, reduzindo de $8,7 \%$ para $3,5 \%$. Por outro lado, a frequência relativa de crianças com história de amamentação exclusiva aumentou, passando de 11,1\% para $16,8 \%$ entre os anos estudados.

Com relação à duração do aleitamento materno, contou-se apenas com os menores que apresentavam um histórico de amamentação, ou seja, que já não mais mamavam no momento da entrevista. Considerando comparativamente os dois anos dos inquéritos, houve uma redução de dez pontos percentuais $(37,1 \%$ para $27,5 \%)$ das crianças que foram alimentadas ao seio por menos de 60 dias e um aumento percentual de valor equivalente para aquelas que mamaram por mais de 180 dias, elevando-se de 19,4\% para 29,6\%. No conjunto dos dados sobre tipologia e duração da amamentação, as diferenças foram estatisticamente significantes $(p<0,001)$. 
Tabela 1. Características biológicas, sociodemográficas, obstétricas e relativas aos serviços de saúde de menores de dois anos e suas mães. (Pernambuco, 1997 e 2006).

\begin{tabular}{|c|c|c|c|}
\hline $\begin{array}{l}\text { Especificação } \\
\text { dos resultados }\end{array}$ & $\begin{array}{c}\text { Ano } 1997 \\
\text { N (\%) }\end{array}$ & $\begin{array}{c}\text { Ano } 2006 \\
\mathrm{~N}(\%)\end{array}$ & Valor $\mathrm{p}^{*}$ \\
\hline \multicolumn{4}{|l|}{ Características relativas às crianças } \\
\hline Sexo & & & 0,599 \\
\hline Masculino & $421(48,4)$ & $315(49,8)$ & \\
\hline Feminino & $449(51,6)$ & $318(50,2)$ & \\
\hline Peso ao nascer & & & 0,578 \\
\hline$<2500 \mathrm{~g}$ & $61(7,4)$ & $51(8,2)$ & \\
\hline $2500-<2999 \mathrm{~g}$ & $167(20,3)$ & $114(18,2)$ & \\
\hline$\geq 3000 \mathrm{~g}$ & $596(72,3)$ & $460(73,6)$ & \\
\hline Idade (meses) & & & 0,052 \\
\hline$<6$ & $238(27,4)$ & $145(22,9)$ & \\
\hline $6-11$ & $220(25,3)$ & $150(23,7)$ & \\
\hline $12-<24$ & $412(47,3)$ & $338(53,4)$ & \\
\hline Índice peso/comprimento & & & 0,504 \\
\hline Baixo peso & $17(2,0)$ & $11(1,8)$ & \\
\hline Peso adequado & $730(86,0)$ & $546(88,1)$ & \\
\hline Peso elevado & $102(12,0)$ & $63(10,2)$ & \\
\hline Tipo de amamentação & & & $<0,001$ \\
\hline Nunca mamou & $72(8,7)$ & $22(3,5)$ & \\
\hline AM Misto & $479(57,9)$ & $374(59,8)$ & \\
\hline AM Predominante & $184(22,2)$ & $124(19,8)$ & \\
\hline AM Exclusivo $^{* *}$ & $92(11,1)$ & $105(16,8)$ & \\
\hline Duração do aleitamento materno (dias) & & & $<0,001$ \\
\hline$<60$ & $225(37,1)$ & $92(27,5)$ & \\
\hline $60-\leq 179$ & $264(43,5)$ & $142(42,8)$ & \\
\hline 180 & $118(19,4)$ & $99(29,6)$ & \\
\hline
\end{tabular}

continua

No que se refere às variáveis de diferenciação entre os anos estudados, foram considerados outros 21 agrupamentos de fatores de ordem biológica, demográfica, sócioambiental e de assistência à saúde que poderiam ter se modificado temporalmente. Nesta perspectiva, como se detalha na primeira tabela, a idade das crianças e de suas mães, a escolaridade materna, a renda per capita da família, o espaço geográfico, o número de pessoas das famílias e algumas características de moradia e condições de saneamento apresentaram mudanças estatisticamente significativas entre 1997 e 2006. Também a realização e o número de consultas pré-natais, orientação sobre amamentação e a visita dos agentes de saúde evidenciaram mudanças nas análises dos dois anos comparados.

A análise subsequente (Tabela 2) trata da frequência de hospitalizações, por diversas causas, de menores de 24 meses; o risco de internação, com os respectivos intervalos de confiança; e posterior análise da comparação de resultados nos anos pesquisados.
Na primeira pesquisa, 110 crianças apresentaram episódios de hospitalizações, correspondendo a $12,6 \%$ do total de 870 da amostra. $\mathrm{Na}$ segunda pesquisa, esse percentual aumentou para 15\%, com 95 internados na amostra de 633 menores de dois anos. Com relação ao número de vezes que as crianças foram internadas por todas as causas, também houve um incremento na frequência de internações hospitalares, elevando a ocorrência de $16,5 \%$ para $17,5 \%$.

$\mathrm{Na}$ distribuição de casos de internamentos por causas específicas em 2006, a frequência de hospitalizações por diarreia apresentou notável aumento, passando de 27,8 para $33,9 \%$. Também percebe-se discreta elevação das internações por desnutrição. A proporção de crianças internadas por pneumonia diminuiu 1,5 pontos percentuais de 1997 para 2006, enquanto ocorreu uma redução na frequência das hospitalizações por outras causas (44,2 para $39 \%)$.

Observando o risco de hospitalização de crianças, há um aumento nesses percentuais em todas as causas específicas de 1997 para 2006. No 
Tabela 1. continuação

\begin{tabular}{|c|c|c|c|}
\hline $\begin{array}{l}\text { Especificação } \\
\text { dos resultados }\end{array}$ & $\begin{array}{c}\text { Ano } 1997 \\
\text { N (\%) }\end{array}$ & $\begin{array}{c}\text { Ano } 2006 \\
\text { N (\%) }\end{array}$ & Valor $\mathbf{p}^{*}$ \\
\hline \multicolumn{4}{|l|}{ Características sociodemográficas maternas } \\
\hline Idade materna (anos) & & & $<0,001$ \\
\hline$\leq 20$ & $154(19,1)$ & $114(18,8)$ & \\
\hline 21 a 30 & $424(52,7)$ & $426(70,4)$ & \\
\hline$\geq 31$ & $227(28,2)$ & $65(10,7)$ & \\
\hline Escolaridade (anos) & & & $<0,001$ \\
\hline$\leq 4$ & $410(51,1)$ & $206(34,2)$ & \\
\hline $5-8$ & $228(28,4)$ & $203(33,7)$ & \\
\hline 9 ou mais & $164(20,4)$ & $194(32,2)$ & \\
\hline Número de filhos & & & 0,196 \\
\hline 1 & $276(34,8)$ & $232(38,3)$ & \\
\hline $2-3$ & $354(44,6)$ & $267(44,2)$ & \\
\hline$\geq 4$ & $163(20,6)$ & $106(17,5)$ & \\
\hline Renda per capita (Salário mínimo) ${ }^{* * *}$ & & & $<0,001$ \\
\hline$<0,5$ & $471(59,0)$ & $506(86,2)$ & \\
\hline$\geq 0,5$ & $327(41,0)$ & $81(13,8)$ & \\
\hline Zona & & & $<0,001$ \\
\hline $\mathrm{RMR}^{\mathrm{a}}$ & $277(35,6)$ & $153(25,4)$ & \\
\hline Interior Urbano & $254(32,6)$ & $162(26,8)$ & \\
\hline Interior Rural & $247(31,7)$ & $289(47,8)$ & \\
\hline Número de moradores & & & 0,004 \\
\hline $2-3$ & $152(19,5)$ & $158(26,2)$ & \\
\hline 4 ou mais & $626(80,5)$ & $446(73,8)$ & \\
\hline Paredes do domicílio & & & $<0,001$ \\
\hline Tijolo & $663(85,2)$ & $571(94,5)$ & \\
\hline Taipa (com ou sem reboco) & $67(8,6)$ & $26(4,3)$ & \\
\hline Outras & $48(6,2)$ & $7(1,2)$ & \\
\hline Piso & & & $<0,001$ \\
\hline Cerâmica & $139(17,9)$ & $133(22,0)$ & \\
\hline Cimento & $535(68,8)$ & $443(73,4)$ & \\
\hline Outros & $104(13,4)$ & $28(4,6)$ & \\
\hline Abastecimento de água & & & 0,011 \\
\hline Rede geral (com e sem canalização interna) & $498(64,0)$ & $346(57,2)$ & \\
\hline Outros & $280(36,0)$ & $258(42,8)$ & \\
\hline Tratamento da água ingerida & & & $<0,001$ \\
\hline Sim & $246(31,6)$ & $315(52,1)$ & \\
\hline Não & $532(68,4)$ & $290(47,9)$ & \\
\hline Esgotamento sanitário & & & 0,002 \\
\hline Rede geral & $205(26,3)$ & $212(35,2)$ & \\
\hline Fossa & $300(38,6)$ & $195(33,4)$ & \\
\hline Outros & $273(35,1)$ & $195(33,4)$ & \\
\hline
\end{tabular}

continua

entanto, nas análises não foram identificadas diferenças estatisticamente significativas na comparação desses resultados.

A distribuição e o risco de hospitalizações em função da tipologia e duração da amamentação estão apresentados na Tabela 3. Ao comparar e analisar os riscos pertinentes nos dois anos estudados, ocorreram diferenças estatisticamente significativas entre as categorias "nunca mamou" e "aleitamento materno misto", com os respectivos valores de $\mathrm{p}=0,003$ e 0,019. Com relação à duração do aleitamento materno, a condição tempo de amamentação igual ou superior a 180 dias foi a única que alcançou significação estatística (valor de $p=0,038$ ).

No ano de 1997, o grupo de crianças que nunca mamou apresentou um percentual de 22,2\% de chance de adoecimento e posterior interna- 


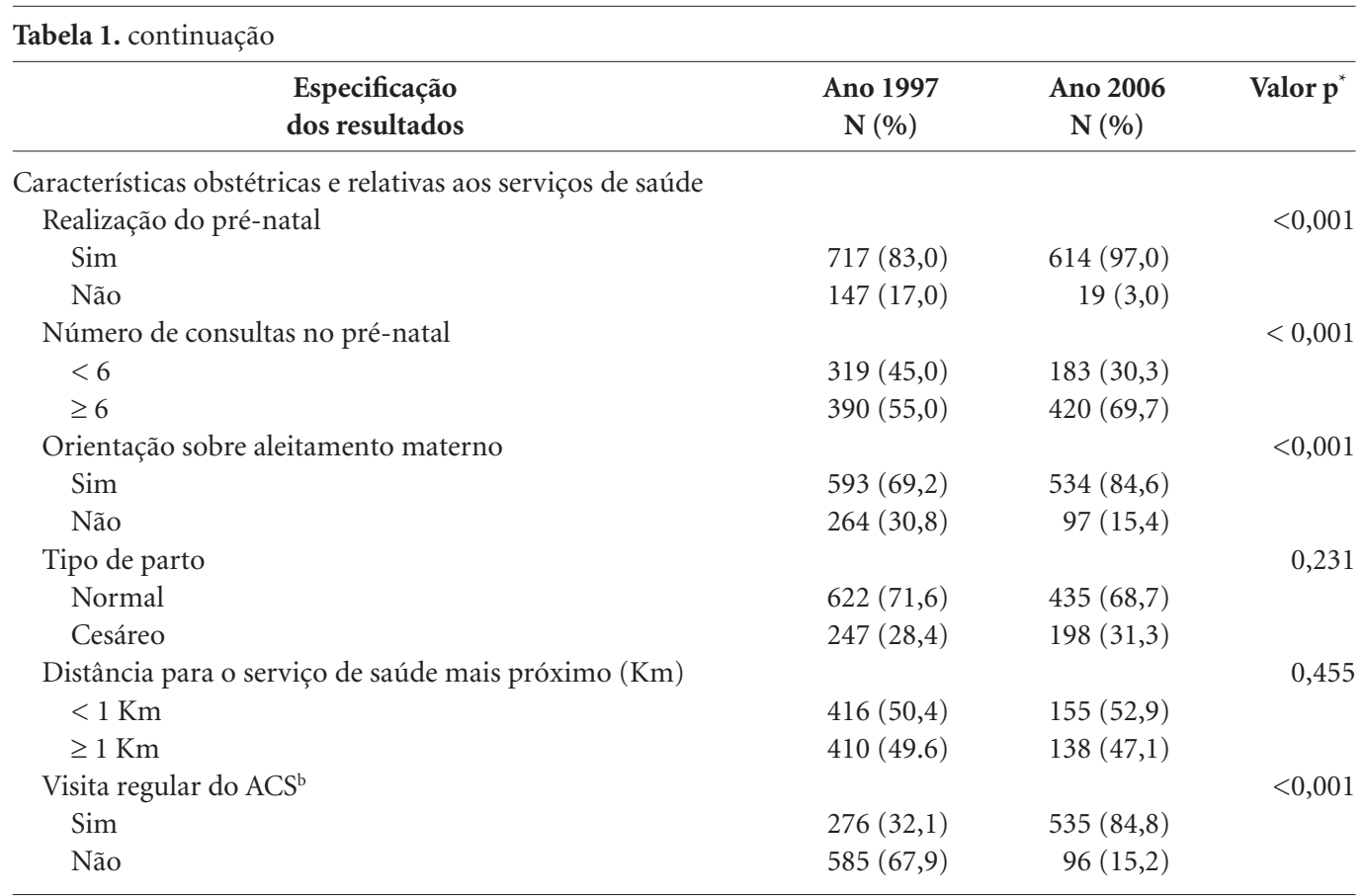

* Teste qui-quadrado de Pearson. ${ }^{* *}$ Considerou-se o aleitamento materno exclusivo de crianças que mamaram apenas no seio até os quatro meses de idade. ${ }^{* * *}$ Salário mínimo em 1997 no valor de $\$ 120,00$ e em 2006 de \$350,00.

a RMR: Região Metropolitana do Recife. ${ }^{b}$ ACS: Agente Comunitário de Saúde.

Tabela 2. Frequência e risco de hospitalização de menores de dois anos por causas, nos 12 meses que antecederam as entrevistas. (Pernambuco, 1997 e 2006).

\begin{tabular}{|c|c|c|c|c|c|}
\hline \multirow{2}{*}{$\begin{array}{c}\text { Causas de } \\
\text { hospitalizações }\end{array}$} & \multicolumn{2}{|c|}{ Ano: $1997(n=870)$} & \multicolumn{2}{|c|}{ Ano: $2006(n=633)$} & \multirow[b]{2}{*}{$\mathbf{p}^{* * *}$} \\
\hline & $\mathbf{N}\left(\%^{*}\right)$ & $\begin{array}{c}\%^{* *} \\
(\text { IC 95\%) }\end{array}$ & $\mathbf{N}\left(\%^{*}\right)$ & $\begin{array}{c}\%^{* *} \\
(\text { IC 95\%) }\end{array}$ & \\
\hline Diarreia & $39(27,8)$ & $4,5(3,2-6,1)$ & $40(33,9)$ & $6,3(4,6-8,5)$ & 0,115 \\
\hline Pneumonia & $33(23,5)$ & $3,8(2,6-5,3)$ & $26(22,0)$ & $4,1(2,7-6,0)$ & 0,757 \\
\hline Desnutrição & $6(4,2)$ & $0,7(0,3-1,5)$ & $6(5,1)$ & $0,9(0,3-2,1)$ & 0,579 \\
\hline Outras causas & $62(44,2)$ & $7,1(5,5-9,0)$ & $46(39,0)$ & $7,3(5,4-9,6)$ & 0,917 \\
\hline Total $1^{\mathrm{a}}$ & $140(16,5)$ & & $118(17,5)$ & & \\
\hline Total $2^{\mathrm{b}}$ & $140(100,0)$ & & $118(100,0)$ & & \\
\hline
\end{tabular}

* Distribuição percentual dos casos de hospitalizações por doenças específicas. ${ }^{* *}$ Percentual de risco de casos de hospitalizações por doenças específicas no conjunto de crianças observadas. ${ }^{* * *}$ Teste qui-quadrado de Pearson. ${ }^{a}$ Total 1: número e percentual de crianças hospitalizadas por todas as causas na amostra de expostos. ${ }^{\text {b }}$ Total 2: número e percentual total em relação aos casos de hospitalizações.

ção. O mais baixo risco $(8,7 \%)$ foi observado nos menores que mamaram exclusivamente ao seio até os quatro meses de vida. Para o ano de 2006, o menor risco de hospitalização manteve-se na categoria das crianças que receberam aleitamento materno exclusivo, com $10,5 \%$ de ocorrência. Já o mais elevado percentual ocorreu nos menores que recebiam aleitamento misto (leite materno e outros tipos de leite). É importante destacar que neste ano, as crianças que nunca mamaram apresentaram uma acentuada redução nos valores de risco (22,2 para 13,6\%) em comparação com 1997, somando-se a um evidente declínio na frequência relativa.

Ainda na Tabela 3, descreve-se a relação de crianças hospitalizadas em função da duração do 
Tabela 3. Distribuição e percentual de risco de hospitalização em função do tipo e duração do aleitamento materno em menores de 24 meses no Estado de Pernambuco, nos anos de 1997 e 2006.

\begin{tabular}{|c|c|c|c|c|c|}
\hline \multirow{3}{*}{ Condição } & \multicolumn{4}{|c|}{ Hospitalizações } & \multirow{3}{*}{$\begin{array}{r}\text { Valor } \\
\mathbf{p}^{* * *}\end{array}$} \\
\hline & \multicolumn{2}{|c|}{ Ano: 1997} & \multicolumn{2}{|c|}{ Ano: 2006} & \\
\hline & $\mathbf{N}\left(\%{ }^{*}\right)$ & $\begin{array}{c}\%{ }^{* *} \\
(\text { IC 95\%) }\end{array}$ & $\mathbf{N}\left(\%{ }^{*}\right)$ & $\begin{array}{c}\%^{* *} \\
(\text { IC 95\%) }\end{array}$ & \\
\hline \multicolumn{6}{|l|}{ Tipo de amamentação } \\
\hline Nunca mamou & $16(15,8)$ & $22,2(13,3-33,6)$ & $3(3,2)$ & $13,6(2,9-34,9)$ & 0,003 \\
\hline AM Misto & $54(53,4)$ & $11,3(8,6-14,4)$ & $63(67,0)$ & $16,8(13,2-21,0)$ & 0,019 \\
\hline AM Predominante & $23(22,8)$ & $12,5(8,1-18,2)$ & $17(18,0)$ & $13,7(8,2-21,0)$ & 0,757 \\
\hline AM Exclusivo & $8(7,9)$ & $8,7(3,8-16,4)$ & $11(11,7)$ & $10,5(5,3-18,0)$ & 0,673 \\
\hline Total & $101(100,0)$ & & $94(100,0)$ & & \\
\hline \multicolumn{6}{|c|}{ Duração do aleitamento materno (dias) ${ }^{* * * *}$} \\
\hline$<60$ & $19(25,0)$ & $8,4(3,6-19,5)$ & $11(22,0)$ & $12,0(6,2-20,6)$ & 0,739 \\
\hline $60-\leq 179$ & $45(59,2)$ & $17,0(12,8-22,2)$ & $19(38,0)$ & $13,4(8,3-20,0)$ & 0,313 \\
\hline$\geq 180$ & $12(15,8)$ & $10,2(5,4-17,1)$ & $20(40,0)$ & $20,2(12,8-29,5)$ & 0,038 \\
\hline Total & $76(100,0)$ & & $50(100,0)$ & & \\
\hline
\end{tabular}

"Distribuição percentual dos casos de hospitalizações segundo o tipo e duração da amamentação. ${ }^{* *}$ Percentual de risco de casos de hospitalizações por tipo e duração da amamentação no conjunto de crianças observadas. ${ }^{* * *}$ Teste Exato de Fisher. ${ }^{* * * *}$ Consideradas apenas as crianças que já haviam encerrado a amamentação.

aleitamento materno, independentemente de sua tipologia. Os menores de dois anos, amamentados por menos de 60 dias, tiveram risco aumentado de internação no ano de 2006, embora não tenha sido estatisticamente significante. Para as crianças que mamaram por mais de 180 dias, o percentual do risco de hospitalizações praticamente dobrou de 1997 para 2006 (10,2\% para 20,2\%), observando significância estatística.

A última tabela apresenta os resultados da análise multivariada através da regressão de Poisson (Tabela 4). Para essa análise, foram selecionadas as variáveis peso ao nascer, índice peso/ comprimento, escolaridade da mãe e número de filhos em função do critério de pré-seleção para compor o modelo (valor de $\mathrm{p}<0,20$ ). Apenas o índice peso/comprimento se manteve como fator de risco de hospitalização de crianças no modelo ajustado $(\mathrm{p}<0,05)$.

\section{Discussão}

Como pontos referenciais para a análise dos resultados em função dos objetivos considerados, algumas observações básicas se impõem: ocorreu entre 1997 e 2006, uma melhoria estaticamente significativa nas práticas do aleitamento materno, como a redução percentual de crianças que nunca mamaram; aumento da frequência dos que usaram o leite materno como fonte exclusiva de alimentação e, como consequência lógica, aumento da duração do aleitamento materno exclusivo por até quatro meses ou mais. Um segundo ponto de destaque consiste em assinalar que, no período analisado, ocorreu discreta mas sistemática elevação das taxas de internações hospitalares das crianças em todos os itens de doenças observadas, embora sem alcançar níveis de significação estatística. Um terceiro aspecto refere-se ao fato de que as variáveis socioeconômicas (exceto renda per capita), demográficas, microambientais, assistência pré-natal, orientação educativa referente ao aleitamento materno e visitas do agente comunitário de saúde, melhoraram de forma expressiva. Por fim, constituindo um foco próprio de abordagem analítica, a verificação de que os resultados da análise bivariada e da regressão logística como explicação estatística dos diversos fatores que contribuíram para a ocorrência das hospitalizações de crianças menores de dois anos em 2006. Esta focalização privilegia a contribuição seletiva das práticas de amamentação.

A evolução favorável da tipologia e duração do aleitamento materno no decurso de nove anos representa um progresso considerável em termos de proteção à saúde das crianças, embora os valores encontrados ainda se distanciem das metas programadas ${ }^{17}$. Na realidade, apesar 
Tabela 4. Razões de prevalências brutas e ajustadas em bloco de fatores para a associação entre hospitalização de menores de dois anos e variáveis biológicas, sociodemográficas, obstétricas e relativas aos serviços de saúde das crianças e suas mães. Estado de Pernambuco, 2006.

\begin{tabular}{|c|c|c|c|c|c|c|}
\hline \multirow{2}{*}{$\begin{array}{l}\text { Especificação } \\
\text { dos resultados }\end{array}$} & \multicolumn{2}{|c|}{ Hospitalizações } & \multirow{2}{*}{$\begin{array}{l}\text { RP bruta } \\
\text { (IC 95\%) }\end{array}$} & \multirow{2}{*}{$\mathbf{p}^{*}$} & \multirow{2}{*}{$\begin{array}{l}\text { RP ajustada } \\
\quad \text { (IC 95\%) }\end{array}$} & \multirow{2}{*}{$\mathbf{p}^{*}$} \\
\hline & Amostra & $\mathrm{N}(\%)$ & & & & \\
\hline \multicolumn{7}{|l|}{ Características relativas às crianças } \\
\hline Sexo & & & & 0,302 & - & \\
\hline Masculino & 315 & $52(16,5)$ & 1,0 & & & \\
\hline Feminino & 317 & $43(13,6)$ & $0,8(0,6-1,2)$ & & & \\
\hline Peso ao nascer & & & & 0,099 & & 0,112 \\
\hline$<2500 \mathrm{~g}$ & 50 & $6(12,0)$ & $0,9(0,4-2,0)$ & & $1,0(0,4-2.1)$ & \\
\hline $2500-<2999 \mathrm{~g}$ & 114 & $24(21,1)$ & $1,6(1,0-2,4)$ & & $1,6(1,0-2,4)$ & \\
\hline$\geq 3000 \mathrm{~g}$ & 460 & $62(13,5)$ & 1,0 & & 1,0 & \\
\hline Idade (meses) & & & & 0,303 & - & \\
\hline$<6$ & 145 & $16(11,0)$ & 1,0 & & & \\
\hline $6-11$ & 150 & $23(15,3)$ & $1,4(0,8-2,5)$ & & & \\
\hline $12-<24$ & 337 & $56(16,6)$ & $1,5(0,9-2,5)$ & & & \\
\hline Índice peso/comprimento & & & & 0,070 & & 0,050 \\
\hline Baixo peso & 11 & $4(36,4)$ & $2,5(1,1-5,7)$ & & $2,7(1,2-6,3)$ & \\
\hline Peso adequado & 545 & $78(14,3)$ & 1,0 & & 1,0 & \\
\hline Peso elevado & 63 & $11(17,5)$ & $1,2(0,7-2,2)$ & & $1,3(0,7-2,3)$ & \\
\hline Tipo de amamentação & & & & 0,433 & - & \\
\hline Nunca mamou & 22 & $3(13,6)$ & $1,3(0,4-4,3)$ & & & \\
\hline AM Misto & 373 & $63(16,8)$ & $1,6(0,9-2,9)$ & & & \\
\hline AM Predominante & 124 & $17(13,6)$ & $1,3(0,6-2,7)$ & & & \\
\hline AM Exclusivo ${ }^{* *}$ & 105 & $11(10,5)$ & 1,0 & & & \\
\hline Duração do aleitamento materno (dias) & & & & 0,216 & - & \\
\hline$<60$ & 101 & $12(11,9)$ & $1,6(0,9-2,8)$ & & & \\
\hline $60-\leq 179$ & 222 & $27(12,2)$ & 1,0 & & & \\
\hline$\geq 180$ & 288 & $53(18,4)$ & $1,3(0,8-2,0)$ & & & \\
\hline
\end{tabular}

continua

do reconhecimento internacional das políticas e programas institucionais do governo brasileiro", e particularmente no caso Pernambuco, os resultados obtidos das últimas três décadas podem ser considerados modestos, traduzindo a resistência cultural (e aqui a cultura é entendida em sua conceituação holística), que se consolidou durante décadas, ou seja, a mercantilização substitutiva dos chamados sucedâneos do leite materno. Foram pelo menos três gerações (avós, mães e filhos em idade reprodutiva) submetidas ao mandato compulsivo do mercado liberal consumista, de modo que a reversão para as condutas saudáveis de amamentação se torna um processo um tanto difícil. Assim, os avanços rápidos relatados em alguns outros estudos são mais a exceção do que a regra ${ }^{18-21}$.

Um outro aspecto, pode revelar um aparente paradoxo: a observação de que a melhoria considerável da situação representada pela evolução positiva de vários indicadores socioeconômicos (exceto salário mínimo, devido ao aumento triplicar no decurso de nove anos), macro e microambientais (localização geográfica, condições físicas das moradias e saneamento), a ampliação do acesso aos serviços de saúde, ao lado da evolução das práticas de aleitamento materno, não tenha se refletido na redução temporal das taxas anuais de hospitalização das crianças, nas comparações entre 1997 e 2006. Conceitualmente, há de se considerar que a disponibilização e, sobretudo, o uso dos serviços de saúde incluindo a assistência hospitalar, como visão descritiva, representam um processo simplificado, mas muitas vezes compreende um processo político e cultural de relativa complexidade. Há poucas décadas, a mortalidade infantil era considerada um evento socialmente aceitável, desejável, portanto culturalmente referendado em várias regiões e países, inclusive no Brasil ${ }^{22}$. Já hoje, é de domínio público a constatação de que as mães e suas famílias acham-se mais motivadas a buscar cuidados mais 
Tabela 4. continuação

\begin{tabular}{|c|c|c|c|c|c|c|}
\hline \multirow{2}{*}{$\begin{array}{c}\text { Especificação } \\
\text { dos resultados }\end{array}$} & \multicolumn{2}{|c|}{ Hospitalizações } & \multirow{2}{*}{$\begin{array}{l}\text { RP bruta } \\
\text { (IC 95\%) }\end{array}$} & \multirow{2}{*}{$\mathbf{p}^{*}$} & \multirow{2}{*}{$\begin{array}{l}\text { RP ajustada } \\
\quad(\text { IC 95\%) }\end{array}$} & \multirow{2}{*}{$\mathbf{p}^{*}$} \\
\hline & Amostra & $\mathrm{N}(\%)$ & & & & \\
\hline \multicolumn{7}{|l|}{ Características sociodemográficas maternas } \\
\hline Idade materna (anos) & & & & 0,310 & - & \\
\hline$\leq 20$ & 114 & $12(10,5)$ & $0,8(0,5-1,3)$ & & & \\
\hline 21 a 30 & 425 & $65(15,3)$ & 1,0 & & & \\
\hline$\geq 31$ & 65 & $12(18,5)$ & $1,1(0,7-1,7)$ & & & \\
\hline Escolaridade (anos) & & & & 0,125 & & 0,112 \\
\hline$\leq 4$ & 206 & $29(14,1)$ & $0,7(0,4-1,1)$ & & $0,7(0,4-1,2)$ & \\
\hline $5-8$ & 203 & $23(11,3)$ & $0,6(0,4-1,0)$ & & $0,6(0,4-1,0)$ & \\
\hline 9 ou mais & 194 & $36(19,3)$ & 1,0 & & 1,0 & \\
\hline Número de filhos & & & & 0,174 & & 0,173 \\
\hline 1 & 232 & $27(11,7)$ & 1,0 & & 1,0 & \\
\hline $2-3$ & 262 & $40(15,3)$ & $1,3(0,8-2,0)$ & & $1,3(0,8-2,0)$ & \\
\hline$\geq 4$ & 103 & $20(19,4)$ & $1,6(0,9-2,8)$ & & $1,7(1,0-2,9)$ & \\
\hline Renda per capita (Salário mínimo) & & & & 0,476 & - & \\
\hline$<0,5$ & 505 & $78(15,4)$ & $1,2(0,7-2,3)$ & & & \\
\hline$\geq 0,5$ & 81 & $10(12,3)$ & 1,0 & & & \\
\hline Zona & & & & 0,780 & - & \\
\hline $\mathrm{RMR}^{\mathrm{a}}$ & 153 & $25(16,3)$ & 1,0 & & & \\
\hline Interior Urbano & 162 & $22(13,6)$ & $0,8(0,5-1,4)$ & & & \\
\hline Interior Rural & 289 & $42(14,5)$ & $0,9(0,6-1,4)$ & & & \\
\hline Número de moradores & & & & 0,397 & - & \\
\hline $2-3$ & 158 & $20(12,7)$ & 1,0 & & & \\
\hline 4 ou mais & 446 & $69(15,5)$ & $1,2(0,8-1,9)$ & & & \\
\hline Paredes do domicílio & & & & 0,235 & - & \\
\hline Tijolo & 571 & $81(14,2)$ & 1,0 & & & \\
\hline Taipa (com ou sem reboco) & 26 & $6(23,1)$ & $1,6(0,8-3,4)$ & & & \\
\hline Outras & 7 & $2(28,6)$ & $2,0(0,6-6,6)$ & & & \\
\hline Piso & & & & 0,477 & - & \\
\hline Cerâmica & 133 & $16(12,0)$ & 1,0 & & & \\
\hline Cimento & 443 & $70(15,8)$ & $1,3(0,8-2,2)$ & & & \\
\hline Outros & 28 & $3(10,7)$ & $0,9(0,3-2,9)$ & & & \\
\hline Abastecimento de água & & & & 0,814 & - & \\
\hline $\begin{array}{l}\text { Rede geral (com e sem canalização } \\
\text { interna) }\end{array}$ & 346 & $52(15,0)$ & 1,0 & & & \\
\hline Outros & 258 & $37(14,3)$ & $1,0(0,6-1,4)$ & & & \\
\hline Tratamento da água ingerida & & & & 0,866 & - & \\
\hline Sim & 315 & $32(10,1)$ & 1,0 & & & \\
\hline Não & 289 & $57(19,6)$ & $1,4(1,0-2,1)$ & & & \\
\hline Esgotamento sanitário & & & & 0,286 & - & \\
\hline Rede geral & 212 & $32(15,1)$ & 1,0 & & & \\
\hline Fossa & 195 & $34(17,4)$ & $1,2(0,7-1,8)$ & & & \\
\hline Outros & 195 & $23(11,8)$ & $0,8(0,5-1,3)$ & & & \\
\hline Destino do Lixo & & & & 0,936 & - & \\
\hline Coleta pública & 362 & $53(14,6)$ & 1,0 & & & \\
\hline Outros & 242 & $36(14,9)$ & $1,0(0,7-1,5)$ & & & \\
\hline
\end{tabular}

continua

extensivos e intensivos de saúde, como o recurso da hospitalização de seus filhos, em etapas ainda precoces (e, portanto, menos graves) do processo patogênico.
Diversos fatores poderiam explicar a maior demanda aos serviços hospitalares, principalmente no nível de assistência pediátrica. As maiores facilidades de acesso aos serviços de saúde, con- 
Tabela 4. continuação

\begin{tabular}{|c|c|c|c|c|c|c|}
\hline \multirow{2}{*}{$\begin{array}{l}\text { Especificação } \\
\text { dos resultados }\end{array}$} & \multicolumn{2}{|c|}{ Hospitalizações } & \multirow{2}{*}{$\begin{array}{l}\text { RP bruta } \\
\text { (IC 95\%) }\end{array}$} & \multirow{2}{*}{$\mathbf{p}^{*}$} & \multirow{2}{*}{$\begin{array}{l}\text { RP ajustada } \\
\quad(\text { IC 95\%) }\end{array}$} & \multirow{2}{*}{$\mathbf{p}^{*}$} \\
\hline & Amostra & $\mathbf{N}(\%)$ & & & & \\
\hline \multirow{2}{*}{\multicolumn{7}{|c|}{$\begin{array}{l}\text { Características obstétricas e relativas } \\
\text { aos serviços de saúde }\end{array}$}} \\
\hline & & & & & & \\
\hline Realização do pré-natal & & & & 0,589 & - & \\
\hline Sim & 613 & $93(15,2)$ & 1,0 & & & \\
\hline Não & 19 & $2(10,5)$ & $0,7(0,1-2,6)$ & & & \\
\hline Número de consultas no pré-natal & & & & 0,491 & - & \\
\hline$<6$ & 182 & $25(13,7)$ & $1,1(0,7-1,7)$ & & & \\
\hline$\geq 6$ & 420 & $67(16,0)$ & 1,0 & & & \\
\hline Orientação sobre aleitamento materno & & & & 0,451 & - & \\
\hline $\operatorname{Sim}$ & 533 & $82(15,4)$ & 1,0 & & & \\
\hline Não & 97 & $12(12,4)$ & $0,8(0,4-1,4)$ & & & \\
\hline Tipo de parto & & & & 0,207 & - & \\
\hline Normal & 434 & $60(13,8)$ & 1,0 & & & \\
\hline Cesáreo & 198 & $35(17,7)$ & $1,2(0,8-1,8)$ & & & \\
\hline $\begin{array}{l}\text { Distância para o serviço de saúde mais } \\
\text { próximo }(\mathrm{Km})\end{array}$ & & & & 0,289 & - & \\
\hline$<1 \mathrm{Km}$ & 155 & $31(20,0)$ & 1,0 & & & \\
\hline$\geq 1 \mathrm{Km}$ & 138 & $21(15,2)$ & $0,7(0,4-1,2)$ & & & \\
\hline Visita regular do $\mathrm{ACS}^{\mathrm{b}}$ & & & & 0,296 & - & \\
\hline Sim & 535 & $84(15,7)$ & 1,0 & & & \\
\hline Não & 96 & $11(11,5)$ & $0,7(0,4-1,3)$ & & & \\
\hline
\end{tabular}

" Poisson. ${ }^{* *}$ Considerou-se o aleitamento materno exclusivo de crianças que mamaram apenas no seio até os quatro meses de idade. ${ }^{* * *}$ Foram inclusas as crianças que ainda estavam sendo amamentadas. ${ }^{a}$ RMR: Região metropolitana do Recife. ${ }^{\mathrm{b}}$ ACS: Agente comunitário de saúde.

tando com expansão da disponibilidade de leitos hospitalares $^{23}$, maior proximidade física desses serviços e a própria aceitação cultural da hospitalização, em fases ainda iniciais da evolução clínica das doenças, poderiam justificar em grande parte esta interpretação. Essa conduta seria também favorecida pelo notável aumento do nível de escolaridade materna e a diminuição nas taxas de fecundidade ao longo do tempo, reduzindo o número de filhos por mães ${ }^{24}$, e, assim, a disposição para incorporar maiores cuidados de saúde às crianças, inclusive as ações de internamentos verificadas no último ano do inquérito (2006).

Estas explicações seriam compatíveis, entre outras, com o fato de que no período observado, reduziu substancialmente a mortalidade infantil e pré-escolar no Estado de Pernambuco ${ }^{25}$, ficando perceptível que o discreto aumento na tendência de internação de crianças não expressa, em princípio, um agravamento na situação de saúde da população infantil, podendo até mesmo ser uma evidência em contrário. É notória a hipótese de mais hospitalizações refletir também mais cuidados de aporte e procura de serviços de saúde retrata uma abordagem fundamentalmente qualitativa, escapando, portanto, dos objetivos e, sobretudo, dos métodos utilizados neste estudo.

Ademais, um dos focos temáticos consiste em analisar a possível influência das práticas de amamentação nos desfechos representados pelo histórico de hospitalizações das crianças. Neste sentido, é pertinente considerar que, em 1997, o maior risco de hospitalização (quase três vezes mais) foi registrado nas crianças que nunca mamaram, em relação às que tiveram aleitamento materno exclusivo; enquanto em 2006, mesmo sem alcançar uma razão de prevalência estatisticamente significativa, esta tendência se manteve. Evidencia-se que as duas condições podem ser consideradas como eventos raros, no sentido de que revelam números amostrais de pequena expressão, sobretudo em 2006, quando apenas 22 menores figuraram no grupo dos que nunca mamaram, dos quais três chegaram a ser hospitalizados. Do mesmo modo, os casos que se mantinham em amamentação exclusiva aos quatro meses de vida e que chegaram a hospitalizar-se foram apenas oito em 1997 e 11 em 2006. Res- 
salta-se ainda que estas situações representaram nos dois anos avaliados, os riscos comparativos mais baixos de internações. Assim, mesmo sem suportes amostrais mais robustos, os resultados acham-se conformados na lógica das hipóteses e sua fundamentação empírica, ou seja, o reconhecimento da função protetora do aleitamento materno contra as mais diversas doenças, principalmente de natureza infecciosa ${ }^{26}$.

Destaca-se ainda que, sem considerar os avanços temporais recentes na mudança dos fatores relacionados à hospitalização de crianças no Estado de Pernambuco e no Brasil ${ }^{27,28}$, ainda permanece o marcante predomínio das diarreias e das pneumonias como principais causas de hospitalização. Convém discutir que o grupo "outras causas de hospitalizações" agregando um complexo diversificado de situações clínicas deve constituir, por sua expressão numérica um objeto adicional de tratamento em outros estudos de campo, destacando a identidade própria de doenças agrupadas nesta categoria. Isto implicaria na conveniência de desagregação dos diversos itens de causas que compõem este grupo.

Configurada na regressão logística, a análise conjunta dos diferentes fatores associados à hospitalização demonstrou que, apesar do considerável número de fatores identificados nas análises univariadas (18 dentre os 23 itens analisados), apenas a relação peso/comprimento, representado pelo baixo peso, obteve resultado estatisticamente significante, mostrando-se associada ao desfecho, coincidindo com resultados de outros estudos descritos na literatura ${ }^{29-31}$. O estado nutricional que reflete essa relação, interfere diretamente na resistência da criança, ou seja, na sua imunocompetência ${ }^{32}$, de modo que crianças desnutridas estariam mais propensas a adoecer e agravar sua condição patológica justificando a necessidade de uma internação.

É oportuno reconhecer as limitações mais evidentes deste estudo. Trata-se da utilização de um banco de dados de duas pesquisas com objetivos próprios e não, como seria ideal, de um projeto concebido e aplicado para responder explicitamente a questão básica referida na introdução do artigo. Isto significa que a amostra como parte da metodologia, inclusive os instrumentos de análise, não puderam ser desenvolvidos em toda a plenitude de uma pesquisa delimitada e específica.

Nesta ordem de apreciação, o aspecto mais limitante passa a ser o da amostra, dificultando as inferências internas (hipóteses de associações) e externas, ou seja, extrapolação dos resultados para um universo populacional mais abrangente. Evidencia-se que a estratificação dos fatores para testar hipóteses reduz a chance dentro dos critérios estatísticos (intervalos de confiança e níveis de significação) de se encontrar diferenças estatísticas nas análises univariadas e, como desdobramento, nas análises multivariadas aqui representadas na regressão logística.

Na realidade, sendo várias as causas de internação, diferentes em cada contexto geográfico e temporal, as interferências que justificam o tratamento hospitalar, reforçam-se na explicação de que, em seu pioneirismo, o relato aqui apresentado, sugere uma perspectiva de pesquisa com um bom potencial de desenvolvimento, a partir dos resultados analisados e, sobretudo, das limitações que relativizam suas possíveis conclusões.

\section{Colaboradores}

TTAG Mota, MFC Caminha, PIC Lira e M Batista Filho trabalharam na concepção, delineamento, análise e interpretação dos dados, redação do artigo, revisão crítica e aprovação da versão a ser publicada. JN Figueiroa trabalhou na construção do arquivo de dados e na análise estatística. 


\section{Referências}

1. Bosi MLM, Machado MT. Amamentação: um resgate histórico. Cad ESP - Esc Saúde Pública do Ceará 2005; 1(1).

2. Barness LA. History of infant feeding practices. Am J Clin Nutr 1987; 46(Supl. 1):168-170.

3. Toma TS, Rea MF. Benefícios da amamentação para a saúde da mulher e da criança : um ensaio sobre as evidências. Cad Saude Publica 2008; 24(2):235-246.

4. Schmid S, Huber D, Hummel M. Early infant feeding and risk of developing type 1 diabetes - associated autoantibodies. JAMA - Am Med Assoc 2003; 290(13): 1721-1728.

5. Dewey K. Is breastfeeding protective against child obesity? J Hum Lact 2003; 19(1):9-18.

6. Owen CG, Whincup PH, Odoki K, Gilg JA CD. Infant feeding and blood cholesterol: a study in adolescents and a systematic review. Pediatrics 2002; 110(3):597-608.

7. Horta BL, Bahl R, Martines JC, Victora CG. Evidence on the long-term effects of breastfeeding: systematic reviews and meta-analyses. Geneva: World Health Organization; 2007.

8. American Academy of Pediatrics. Breastfeeding and the use of human milk. Pediatrics 2005; 115(2):496-506.

9. Brasil. Ministério da Saúde (MS). Secretaria de Atenção à Saúde. Departamento de Atenção Básica. Saúde da criança: Nutrição Infantil. Aleitamento Materno e Alimentação Complementar. Brasília: Editora MS; 2009. (Série A. Normas e Manuais Técnicos. Cadernos de Atenção Básica, no 23).

10. Rea MF, Araújo MDFM de. Implementation of breast-feeding practices in Brazil: From international recommendations to local policy. In: Freire WB, editor. Nutrition and an active life: from knowledge to action. Washington: Pan American Health Organization; 2005. Scientific and Technical Publication (612). p. 109-130.

11. Fundo das Nações Unidas para a Infância (Unicef). Crianças e adolescentes em Pernambuco: saúde, educação e trabalho. I Pesquisa Estadual de Saúde e Nutrição. Brasília: Unicef; 1992.

12. Pernambuco. Secretaria de Saúde (SSPE). Universidade Federal de Pernambuco (UFPE). Departamento de Nutrição. Instituto de Medicina Integral Professor Fernando Figueira (IMIP). II Pesquisa Estadual de Saúde e Nutrição: Saúde, Nutrição, Alimentação e Condições Socioeconômicas no Estado de Pernambuco. Recife: SSPE, UFPE, IMIP; 1998.

13. Pernambuco. Secretaria de Saúde (SSPE). Universidade Federal de Pernambuco (UFPE). Departamento de Nutrição. Instituto de Medicina Integral Professor Fernando Figueira (IMIP). III Pesquisa Estadual de Saúde e Nutrição: Situação Alimentar, Nutricional e de Saúde no Estado de Pernambuco. Relatório Final. Recife: SSPE, UFPE, IMIP; 2007.

14. World Health Organization (WHO). Indicators for assessing infant and young child feeding practices: conclusions of consensus meeting held 6-8 November 2007 in Washington D.C., USA. Washington: WHO; 2007.

15. Carmo EH, Barreto ML, Silva Júnior JB. Mudanças nos padrões de morbimortalidade da população brasileira: os desafios para um novo século. Epidemiol e Serviços Saúde 2013; 12(2):63-75.

16. Brasil. Ministério da Saúde (MS). Conselho Nacional de Saúde. Resolução no 466, de 12 de dezembro de 2012. Diário Oficial da União 2013; 13 jun.
17. World Health Organization (WHO). Global Strategy for Infant and Young Child Feeding. Geneva: WHO; 2003.

18. Ceron DK, Lazzaretti FO, Migott AMB, Geib LTC. Efeito das ações de promoção do aleitamento na duração da amamentação em duas filiações maternas. Rev Eletrônica Enferm 2012; 14(2):345-354.

19. Caminha M de FC, Filho MB, Serva VB, Arruda IKG de, Figueiroa JN, Lira PIC. Tendências temporais e fatores associados à duração do aleitamento materno em Pernambuco. Rev Saude Publica 2010; 44(2):240-248.

20. Saliba NA, Zina LG, Moimaz, Suzely Adas Saliba Saliba $O$. Freqüência e variáveis associadas ao aleitamento materno em crianças com até 12 meses de idade no município de Araçatuba, São Paulo, Brasl. Rev Bras Saúde Matern Infant Recife 2008; 8(4):481-490.

21. Rea MF. Reflexões sobre a amamentação no Brasil : de como passamos a 10 meses de duração. Cad Saude Publica 2003; 19(Supl. 1):37-45.

22. Vailati LL. Representaçoes da morte infantil durante o século XIX no Rio de Janeiro e na Inglaterra: Um esboço comparativo preliminar. Rev Hist (Costa Rica) 2012; 167:261-294.

23. Porto SM, Santos IS, Ugá MAD. A utilização de serviços de saúde por sistema de financiamento. Cien Saude Colet 2006; 11(4):895-910.

24. Berquó E, Cavenaghi S. Fecundidade em declínio: Breve nosta sobre a reduçao no número médio de filhos por mulher no Brasil. Novos Estud 2006; 74:11-15.

25. Vidal SA, Frias PG de, Barreto FMP, Vanderlei LCM, Felisberto E. Óbitos infantis evitáveis em hospital de referência estadual do Nordeste brasileiro. Rev Bras Saúde Matern Infant 2003; 3(3):281-289.

26. Village EG. Breastfeeding and the Use of Human Milk. Pediatrics 1997; 100(6):1035-1039.

27. Castro MSM. Desigualdades sociais no uso de internações hospitalares no Brasil : o que mudou entre 1998 e 2003. Cien Saude Colet 2003; 11(4):987-998.

28. Ferrer APS, Sucupira ACSL, Grisi SJFE. Clinical science causes of hospitalization among children ages zero to nine years old in the city of São Paulo, Brazil. Clinics 2010; 65(1):35-44.

29. Victora CG, Barros FC, Kirkwood BR, Vaughan JP. Pneumonia, diarrhea, and growth in the first 4 of life: a longitudinal study of 5914 urban Brasilian chikdren. Am J Clin Nutr 1990; 52(2):391-396.

30. Nascimento LFC, Marcitelli R, Agostinho FS, Gimenes CS. Análise hierarquizada dos fatores de risco para pneumonia em crianças. J Bras Pneumol 2004; 30(5):445-451.

31. Vanderlei LCM, Silva GAP da. Diarréia aguda : o conhecimento materno sobre a doença reduz o número de hospitalizações nos menores de dois anos? Rev Assoc Med Bras 2004; 50(3):276-281.

32. Monte CMG. Desnutrição : um desafio secular à nutrição infantil. J Pediatr (Rio J) 2000; 76(Supl. 3):285-297.

Artigo apresentado em 02/09/2014

Aprovado em 26/01/2015

Versão final apresentada em 28/01/2015 